\title{
The transcriptome of lae 1 mutants of Trichoderma reesei cultivated at constant growth rates reveals new targets of LAE1 function
}

\author{
Erzsébet Fekete ${ }^{1}$, Levente Karaffa ${ }^{1 *}$, Razieh Karimi Aghcheh², Zoltán Németh ${ }^{1}$, Éva Fekete ${ }^{1}$, Anita Orosz ${ }^{1}$,
} Melinda Paholcsek ${ }^{3}$, Anikó Stágel ${ }^{4}$ and Christian P Kubicek ${ }^{5}$

\begin{abstract}
Background: The putative methyltransferase LaeA is a global regulator that affects the expression of multiple secondary metabolite gene clusters in several fungi. In Trichoderma reesei, its ortholog LAE1 appears to predominantly regulate genes involved in increasing competitive fitness in its environment, including expression of cellulases and polysaccharide hydrolases. A drawback in all studies related to LaeA/LAE1 function so far, however, is that the respective loss-of-function and overexpressing mutants display different growth rates. Thus some of the properties attributed to LaeA/LAE1 could be simply due to changes of the growth rate.

Results: We cultivated T. reesei, a $\Delta$ lae 1 mutant and a lae1-overexpressing strain in chemostats on glucose at two different growth rates $\left(0.075\right.$ and $\left.0.020 \mathrm{~h}^{-1}\right)$ which resemble growth rates at repressing and derepressing conditions, respectively. Under these conditions, the effect of modulating LAE1 expression was mainly visible in the $\Delta$ lae1 mutant, whereas the overexpressing strain showed little differences to the parent strain. The effect on the expression of some gene categories identified earlier (polyketide synthases, heterokaryon incompatibility proteins, PTH11-receptors) was confirmed, but in addition GCN5-N-acetyltransferases, amino acid permeases and flavin monooxygenases were identified as so far unknown major targets of LAE1 action. LAE1 was also shown to interfere with the regulation of expression of several genes by the growth rate. About a tenth of the genes differentially expressed in the 4 lael mutant under either growth condition were found to be clustered in the genome, but no specific gene group was associated with this phenomenon.
\end{abstract}

Conclusions: Our data show that - using T. reesei LAE1 as a model - the investigation of transcriptome in regulatory mutants at constant growth rates leads to new insights into the physiological roles of the respective regulator.

Keywords: Trichoderma reesei, Microarray, Chemostat, LAE1, Growth rate, Transcriptome

\section{Background}

Reverse genetics relies on the use of molecular biological methods to discover the function of a gene by analyzing the phenotypic effects that result from a manipulation of its function. While this approach mostly leads to valid information about the role of a gene in the physiology of the respective organism, some caveats are to be applied when these mutants are studied by -omics techniques: many gene mutants also display altered growth rates,

\footnotetext{
* Correspondence: karaffa.levente@science.unideb.hu

'Department of Biochemical Engineering, Faculty of Science and Technology, University of Debrecen, Egyetem tér 1, H-4032 Debrecen, Hungary

Full list of author information is available at the end of the article
}

and thus studying their gene or protein expression on plates or in batch cultures will inevitably also identify genes whose expression is controlled only by the growth rate itself. This may lead to flawed interpretations as to the potential targets of the investigated genes, particularly in the case of regulatory genes.

The Aspergillus nidulans LaeA protein, a putative $S$-adenosylmethionine-dependent (SAM) methyltransferase, was originally described as a global regulator of secondary metabolism [1]. LaeA is known to be a nuclear protein, and is localized to the nucleus [1-3]. It has therefore been suggested that it regulates transcription by protein lysine or protein arginine methyltransferase functions [1], and its 
function has putatively been linked to changes in chromatin structure [4]. Recent studies in A. nidulans, however, failed to identify a protein that is methylated by LaeA, but considerable automethylation was observed [5]. In addition, the LAE1- (the Trichoderma reesei orthologue of Aspergillus LaeA) effected transcriptome of Trichoderma reesei showed no correlation with histone methylation at the affected loci [6].

LaeA was later shown to also control developmental events, such as conidiation in numerous fungi [7-13], and fruiting body formation in A. flavus [14,15]. We have recently shown that in T. reesei, LAE1 also controls conidiation, the expression of polysaccharide hydrolytic enzymes and of genes involved in eco-physiological fitness, whereas the effect on secondary metabolite biosynthesis was not that strongly pronounced as in the Aspergilli $[16,6]$. Thus, current knowledge suggests that LaeA has a dynamic role in both fungal morphological and chemical development, and nutrition.

In $T$. reesei, lae1 mutants are also differing in their growth rates, being slower in the loss-of-function mutant and faster in mutants overexpressing lae1 [16]. We thus wondered to which extent our recent study on the LAE1-effected transcriptome of $T$. reesei [6] would have been biased by the different growth rates of the lae1 mutants. This could have been particularly due for genes that are known to be expressed only at specific growth rates, such as e.g. those involved in secondary metabolite biosynthesis. Therefore, we chose to use chemostat cultures on D-glucose as a carbon source at two different growth rates (one carbon catabolite repressing and one carbon catabolite derepressing) to investigate the genomewide changes in gene expression in relation to LAE1 function, using $\Delta l a e 1$ and OElae1 recombinant mutant strain of $T$. reesei.

\section{Results}

\section{Verification of the experimental strategy}

Constant-mass, carbon-limited, chemostat-type continuous fermentations were used to cultivate the parent strain T. reesei QM 9414, and the Slae1 and OElae1 mutant strains derived from it on glucose $(1 \%, \mathrm{w} / \mathrm{v})$ as a sole carbon source at two different dilution rates $\left(D=0.075 \mathrm{~h}^{-1}\right.$ and $\mathrm{D}=0.020 \mathrm{~h}^{-1}$ ). These two dilution rates (henceforth referred to as "high" and "low" growth rate) have previously been shown to represent a state of carbon catabolite repression and of carbon catabolite derepression, respectively, in T. reesei and also in A. nidulans [17-19]. Cultures were grown batchwise for 24 hours after inoculation. At the first 6-7 residence times of the continuous cultivations, gradually attenuating oscillation of the specific biomass production occurred [20] after which the oscillation decreased to a non-significant level. The steady-state biomass concentration was $1.49 \pm 0.11 \mathrm{~g} / \mathrm{L}$ in all cultures irrespective of the dilution rate. By feeding the cultures with a medium containing $3 \mathrm{~g} / \mathrm{L}$ D-glucose as a sole carbon source (see Methods section), this biomass data resulted in a calculated growth yield (grams of biomass formed per gram of carbon source consumed) of between 46 and 53\% for all cultures, which correlates well with previously published data from various fungal D-glucose-limited cultures (see [20] for references), and with our previous studies on $T$. reesei steady-states $[17,18]$. The residual glucose concentration was between 0.03 and $0.05 \mathrm{mM}$ at both 0.075 and $0.020 \mathrm{~h}^{-1}$. These values correlate well with the affinities of the high affinity hexose transporters of filamentous fungi [21], and thus prove that our cultures were indeed glucose-limited. At $0.02 \mathrm{~h}^{-1}$ the low dilution rate, some conidiospore formation was visible in the parent strain but not at the $\Delta$ lae1-mutant. In addition, both strains exhibited a small degree of pellet formation although the overwhelming majority of cells displayed filamentous morphology at $0.075 \mathrm{~h}^{-1}$. In summary, while there were some minor morphological differences between the two strains at the two dilution rates applied, they unlikely affected the general experimental strategy. We thus considered the system appropriate for the purpose of this study.

\section{LAE1 loss of function is more dominant at high growth rates}

1069 of the 9123 genes that were used in the array showed an at least 2 -fold up- or downregulation (with $\mathrm{p}<0.05$ ) in T. reesei $\Delta$ lae1 when compared to the parent strain QM 9414 (Table 1; Additional file 1: Table S1). Of these, only 182 genes were $>2$-fold regulated both at 0.075 and $0.02 \mathrm{~h}^{-1}$, respectively. The highest number of genes was encountered

Table 1 Number of genes affected by lae1 manipulation in T. reesei at the two growth rates in comparison to the parent strain QM 9414

\begin{tabular}{|c|c|c|c|c|c|c|c|c|}
\hline \multirow{2}{*}{ Strain comparison } & \multirow[t]{2}{*}{$\mathrm{D}\left[\mathrm{h}^{-1}\right]$} & \multirow[t]{2}{*}{ Total } & \multicolumn{3}{|c|}{ Upregulated } & \multicolumn{3}{|c|}{ Downregulated } \\
\hline & & & All genes* & Orphan genes & Unknown genes & All genes* & Orphan genes & Unknown genes \\
\hline Slael vs. QM 9414 & 0.075 & \multirow{2}{*}{1069} & 523 & 33 & 193 & 407 & 16 & 138 \\
\hline Dlae1 vs. QM 9414 & 0.020 & & 203 & 10 & 79 & 118 & 9 & 40 \\
\hline OElael vs. QM 9414 & 0.075 & \multirow{2}{*}{113} & 53 & & 22 & 36 & 1 & 13 \\
\hline OElael vs. QM 9414 & 0.020 & & 11 & & 4 & 17 & 2 & 3 \\
\hline
\end{tabular}

*including unknown and orphan genes. 
when the $\Delta$ lae1 mutant was compared to the parent strain QM9414 at $0.075 \mathrm{~h}^{-1}$ (930 vs. 321 genes at high vs. low growth rate). Genes that were downregulated in the $\Delta$ lae1 strain accounted for the minor fraction in both cases $(43 \%$ and $36 \%$ at high and low growth rates, respectively).

Only a considerably smaller number of genes (113) was differently regulated between the OElae1 mutant and the parent strain ( 89 and 28 genes at high and low growth rate, respectively, with 4 genes being affected at both growth rates). Genes that were downregulated accounted for a minor fraction at the high growth rate, but accounted for $61 \%$ at the lower growth rate (Table 1; Additional file 2: Table S2). The validity of the array data was approved by qPCR analysis of the expression of a small number of genes (Additional file 3: Table S3).

Under all conditions (except for OElae1 at low growth rates, but this is likely due to the small gene number), more than a third of all genes encoded unknown proteins (i.e. such that shared orthologs in at least some other Pezizomycota) or were orphan genes (Table 1). In order to assess the global changes in gene expression brought about by the two lae1 mutations, those genes for which a function was known or can be predicted were classified according to the FunCat categorization [22]; Figure 1. Of these, the majority of genes belonged to category "metabolism", followed by "cellular transport" and "transcription". However, none of the categories exhibited a statistically significant difference over another ( $p>0.05$ in all cases) when the number of genes in the individual categories was related to the total number of genes that were differently expressed under any of these conditions, This indicates that the cellular effect of the two lae1 mutations is not reflected by changes in the expression of one general gene category.

Since the effect of LAE1 modulation may rather be reflected in the expression of genes encoding FunCat subcategories, we calculated the percentage of LAE1effected genes of several subcategories in the complete ( $\geq 2$-fold, $\mathrm{p}<0.05$ ) transcriptome and compared it to the percentage of these subcategories in the whole $T$. reese $i$ genome. Thereby, some significant changes were observed (Table 2): genes encoding amino acid transporters, heteroincompatibility (HET) proteins, GCN5-N-acetyltransferases, and polyketide synthases $(\mathrm{PKS})$ were significantly $(\mathrm{p}<0.05)$ more abundant in the transcriptome affected in $\Delta l a e 1$ at high growth rates than in the genome. A significantly higher number of genes belonging to the first three gene groups (vide supra) were differently expressed at the low growth rate. Genes encoding the PTH11-type receptors were significantly more abundant among genes that are higher expressed in the $\Delta$ lae 1 mutant at high growth rates.

In addition, LAE1-effected genes involved in metabolism were found to have a strong bias towards amino acid metabolism at the high growth rate, with the majority of them being downregulated (Figure 2). No significant changes in the expression of genes involved in intermediary metabolism (carbohydrate, lipid, amino acid and nucleotide metabolism) were noted at the low growth rate.

With regards to secondary metabolism, only 3 of the 11 polyketide synthases (PKS), and 3 of the 10 nonribosomal peptide synthases (NRPS) of $T$. reesei were shown to be affected by a loss of LAE1 function (Table 2),

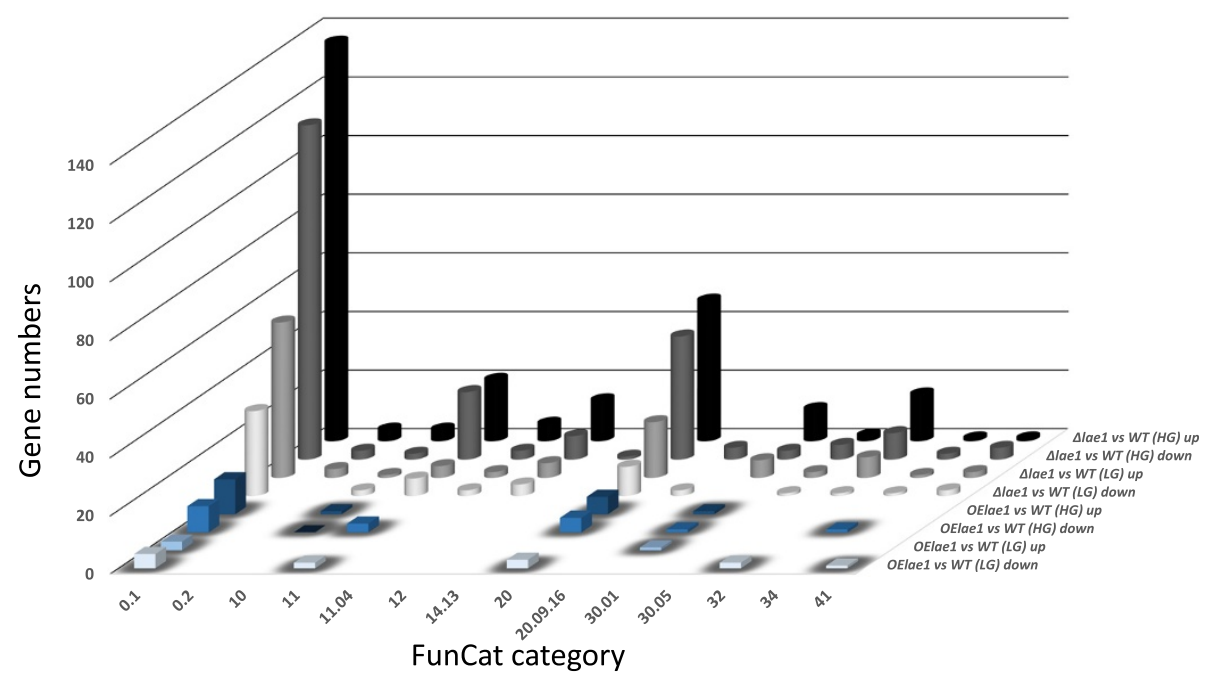

Figure 1 Expression of genes belonging to selected FunCat groups in T. reesei $\triangle$ lae1 (DLAE1) and T. reesei OElae1 (OElae1) during cultivation at $0.075(\mathrm{HG})$ and $0.02(\mathrm{LG}) \mathbf{h}^{-1}$, respectively. "Up" means 2-fold upregulation, "down" 2-fold downregulation when compared to T. reesei QM9414 (WT). FunCat categories shown are: 01, metabolism; 02, energy generation; 10, cell cycle and DNA processing; 11, transcription; 11.4, RNA processing; 12, protein synthesis; 14.13, protein degradation; 20, transport; 20.09.16, cellular export and secretion; 30.01, intracellular signalling; 30.5, transmembrane signaling; 32, cell rescue and defense; 34, interaction with cellular environment; 41, development. 
Table 2 Comparison of some FunCat subcategory genes differentially expressed in the $\Delta / a e 1$ mutant compared to the T. reesei parent strain

\begin{tabular}{|c|c|c|c|c|c|c|c|c|}
\hline \multirow{2}{*}{ Function } & \multirow{2}{*}{ FunCat } & \multicolumn{2}{|c|}{$\mathrm{D}=0.075\left[\mathrm{~h}^{-1}\right]$} & \multicolumn{2}{|c|}{$\mathrm{D}=0.020\left[\mathrm{~h}^{-1}\right]$} & \multicolumn{2}{|c|}{ Present in the genome } & \multirow[t]{2}{*}{ p-value } \\
\hline & & Down & Up & Down & Up & Number & $\%$ & \\
\hline Amino acid transporters & 20_01_07 & 11 & 7 & 2 & 3 & 36 & 0.4 & $<0.01$ \\
\hline $\mathrm{C} 2 \mathrm{H} 2$ transcription factors & 11_02_03_04** & 3 & 6 & 0 & 1 & 54 & 0.6 & \\
\hline Cytochrome P450 proteins & 32_07 & 2 & 6 & 1 & 0 & 61 & 0.7 & \\
\hline Flavin monooxygenases & 16_21_05 & 2 & 5 & 0 & 4 & 43 & 0.5 & \\
\hline GCN5N-acetyltransferases & 14_07 & 5 & 3 & 2 & 1 & 20 & 0.2 & $<0.02$ \\
\hline Glycoside hydrolases & 01_25_01 & 6 & 10 & 0 & 7 & 194 & 2.1 & \\
\hline Heteroincompatibility proteins & 36_20 & 4 & 1 & 1 & 1 & 23 & 0.25 & $<0.05$ \\
\hline Major facilitator superfamily & 20_03 & 16 & 21 & 3 & 11 & 174 & 1.9 & \\
\hline Mitochondrial function & $42 \_16$ & 3 & 3 & 3 & 1 & 83 & 0.9 & \\
\hline NRPS & 01_20_36 & 1 & 2 & 1 & 0 & 10 & 0.11 & \\
\hline PKS & 01_20_05 & 2 & 1 & 0 & 0 & 12 & 0.13 & $<0.05$ \\
\hline PTH11-receptors & 30_05_02_24 & 2 & 3 & 0 & 1 & 24 & 0.25 & \\
\hline SSCRP & $70 \_27$ & 14 & 12 & 0 & 3 & 130 & 1.4 & \\
\hline
\end{tabular}

*Only given for subcategories that were statistically supported to behave differently in the mutant.

**The subcategory specification refers to all transcription factors; $\mathrm{C} 2 \mathrm{H} 2$ transcription factors do not have their own number.

and with the exception of the NRPS Trire2:71005, which was downregulated in the $\Delta$ lae1 mutant at both growth rates, all the other genes were down- or upregulated only at $\mathrm{D}=0.075 \mathrm{~h}^{-1}$. It is interesting to note that this number of affected PKS- and NRPS-encoding genes is nevertheless significantly higher than in our previous study [6], in which only a single NRPS gene (the siderophore synthase
Trire2:69946) was found to be $>2$-fold downregulated in the $\Delta$ lae1 mutant.

We also applied the same analysis to the genes which were at least 2-fold differently regulated between the OElae1 mutant and the parent (Additional file 2: Table S2). However, no specific gene category or subcategory was found to accumulate to a significant extent.

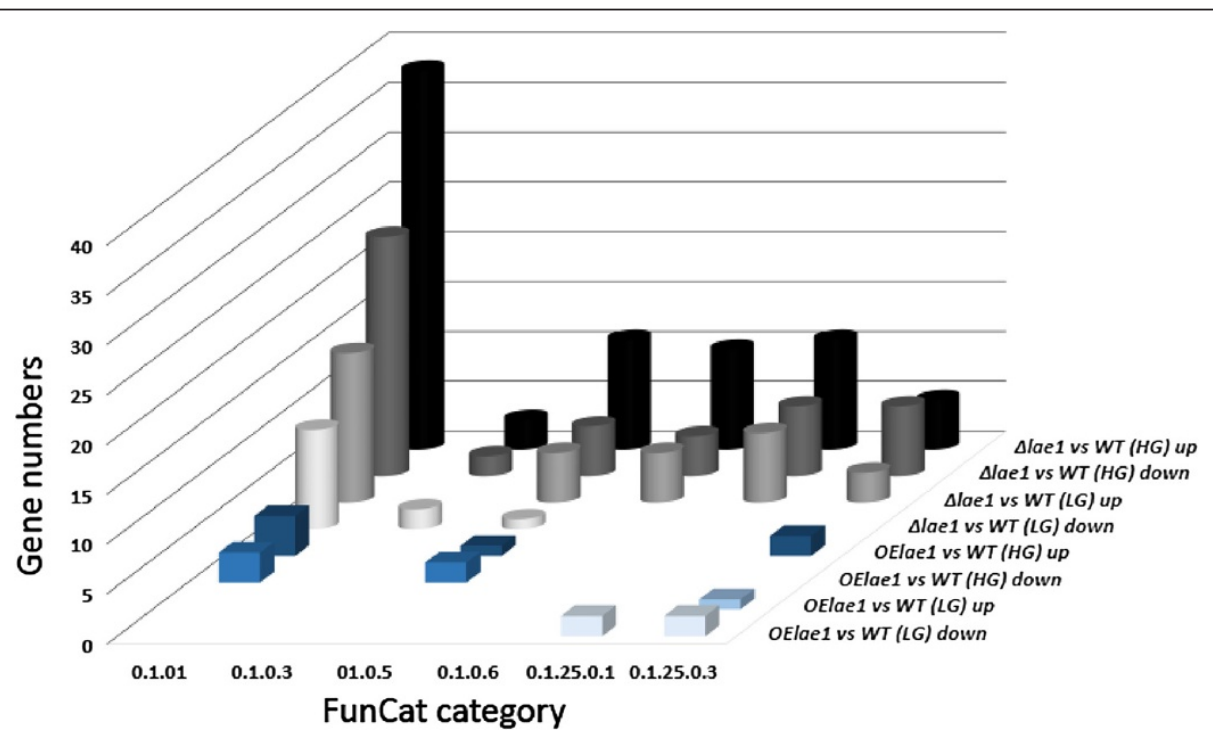

Figure 2 Expression of genes belonging to metabolism-related FunCat groups in T. reesei $\triangle$ lae1 ( $\triangle L A E 1)$ and T. reesei OElae1 (OElae1) during cultivation at $0.075(\mathrm{HG})$ and $0.02(\mathrm{LG}) \mathbf{h}^{-1}$, respectively. "Up" means 2-fold upregulation, "down" 2-fold downregulation when compared to T. reesei QM9414 (WT). FunCat categories shown are: 01.01, amino acid metabolism; 01.03, nucleotide metabolism; 01.05, carbohydrate metabolism; 01.06, lipid metabolism; 01.25.01, extracellular carbohydrate degradation; 01.25.03, extracellular protein degradation. 


\section{LAE1 loss-of-function affects growth rate regulation of gene expression}

While the above data revealed genes that are affected by LAE1 function at a constant growth rate, we surmised that there may be also genes whose response to changes in the growth rate is dependent on LAE1. To this end we retrieved two data sets: one which contained genes that were growth rate dependent in $T$. reesei wild-type, but not in Slae1, and another one which contained only genes whose expression was growth rate dependent in the Alae1 mutant strain, but not in the wild type. This revealed a total of 758 and 131 genes, respectively. Their annotation is given in Additional files 4 and 5: Tables S4 and S5: as can be seen, no specific gene category was significantly enriched in these two gene pools, indicating that the interference of LAE1 with growth-rate regulation affects specific genes rather than gene groups of similar function. A similar analysis was also made for the effect of lae1 overexpression. Again, the number of genes was considerably smaller, and the majority of them consisted of unknown proteins (data not shown).

In addition, we also monitored those genes, whose growth-rate dependent expression in the lae1 mutants was reverted, i.e. opposite as to the parent strain. 26 genes that were higher expressed at the high growth rate in T. reesei QM 9414 were found to be downregulated in $T$. reesei $\Delta l a e 1$, and 12 genes displayed the opposite trend. No specific FunCat category or subcategory was detected to be enriched in these two groups, which accounted for only a minor portion of the LAE1 effected transcriptome (Additional file 6: Table S6).

\section{The majority of genes effected by LAE1 are not clustered} in the genome

We were further interested whether the genes affected in their expression by LAE1 function would show a clustering in the genome. To test this, we made use of REEF, a program that performs a serial hypergeometric distribution tests in a sliding window along the chromosomes implemented in Phyton program [23]. This program has the advantage that it uses statistical models to assess the significance of physical clusters detected. It has also previously shown to lead results consistent with other methods [24]. REEF detected 124 and 31 of the 930 and 321 genes differentially expressed between the $\Delta l a e 1$ and the parent strain during cultivation at high and low growth rate, respectively, to be clustered in the genome (Table 3). The clustering was evident from a 2.2 and 2.6fold enrichment in gene density in the transcripts from high and low growth rates, respectively, over the average

Table 3 Clustering of transcripts that are differentially expressed in $\Delta / a e 1$ vs the parent T. reesei QM 9414 at the two growth rates

\begin{tabular}{|c|c|c|c|c|c|c|c|c|c|}
\hline \multirow{3}{*}{ Scaffold } & \multirow{3}{*}{$\begin{array}{c}\text { Size } \\
\text { [Mbp] }\end{array}$} & \multicolumn{4}{|c|}{$D=0.075\left[h^{-1}\right]$} & \multicolumn{4}{|c|}{$D=0.020\left[h^{-1}\right]$} \\
\hline & & \multicolumn{2}{|c|}{ Area [Mbp] } & \multirow{2}{*}{$\begin{array}{l}\text { Genes } \\
\text { clustered }\end{array}$} & \multirow{2}{*}{$\begin{array}{c}\text { Cluster size } \\
\text { [genes] }\end{array}$} & \multicolumn{2}{|c|}{ Area [Mbp] } & \multirow{2}{*}{$\begin{array}{c}\text { Genes } \\
\text { clustered }\end{array}$} & \multirow{2}{*}{$\begin{array}{c}\text { Cluster size } \\
\text { [genes] }\end{array}$} \\
\hline & & Begin & End & & & Begin & End & & \\
\hline 1 & 2.76 & 2.26 & 2.43 & 9 & 50 & 2.45 & 2.57 & 3 & 39 \\
\hline \multirow[t]{2}{*}{2} & 2 & 0.04 & 0.15 & 7 & 28 & 0.04 & 0.21 & 4 & 44 \\
\hline & & 1.33 & 1.45 & 7 & 30 & & & & \\
\hline 3 & 1.91 & 0 & 0.16 & 9 & 54 & & & & \\
\hline \multirow[t]{2}{*}{4} & 1.83 & 1.08 & 1.19 & 7 & 29 & 0.64 & 0.81 & 4 & 45 \\
\hline & & 1.15 & 1.26 & 7 & 30 & & & & \\
\hline 5 & 1.73 & 0.7 & 0.86 & 8 & 39 & 0.84 & 1.04 & 5 & 61 \\
\hline 6 & 1.45 & 0.41 & 0.54 & 8 & 28 & 0.84 & 1.01 & 3 & 36 \\
\hline \multirow[t]{2}{*}{7} & 1.43 & 1.07 & 1.21 & 9 & 44 & 1.13 & 1.25 & 3 & 37 \\
\hline & & 1.21 & 1.33 & 8 & 31 & & & & \\
\hline \multirow[t]{3}{*}{8} & 1.41 & 0.35 & 0.52 & 9 & 52 & & & & \\
\hline & & 0.57 & 0.69 & 7 & 27 & & & & \\
\hline & & 0.83 & 0.94 & 8 & 35 & & & & \\
\hline 10 & 1.16 & 0.92 & 1.04 & 7 & 28 & & & & \\
\hline 19 & 0.66 & 0.55 & 0.66 & 7 & 27 & 0.5 & 0.61 & 3 & 23 \\
\hline 20 & 0.63 & & & & & 0.43 & 0.55 & 3 & 19 \\
\hline 24 & 0.5 & 0.21 & 0.32 & 7 & 22 & & & & \\
\hline 25 & 0.44 & & & & & 0.16 & 0.3 & 3 & 35 \\
\hline Total: & & & & 124 & 554 & & & 31 & 339 \\
\hline
\end{tabular}


distribution in the genome. 16 and 9 clusters were found for the transcripts from high and low growth rate, of which 4 were present under both conditions. 7 and 5, respectively, occurred with a 200,000 bp distance from one of the ends of the respective scaffold. This demonstrates that about a tenth of the genes affected in their expression by LAE1 loss-of-function are clustered in the genome.

We also evaluated the genes contained in these clusters, but no obvious trend was seen: gene groups more frequently occurring were identical to those more abundant in the genome. We also specifically looked for clustering of genes encoding secondary metabolites (NRPS, PKS) and potentially being involved in their biosynthesis (transcription factors, ABC transporters, cytochrome $\mathrm{P} 450$ proteins, aldo/keto reductases/dehydrogenases, flavoproteins) but obtained a similar result (data not shown).

\section{Discussion}

To investigate the physiology of microbial mutant strains, they are usually cultivated on solid medium or in batch cultures. Under both conditions, individual nutrients will gradually become growth limiting, and cause changes in the actual specific growth rates, that in turn may profoundly influence the metabolism and consequently the physiology of the cell. While this fact has been well considered in studies of yeasts and bacteria, much fewer reports are available for the applications of methods that take the specific growth rate into consideration for filamentous fungal research [17-19,25-28].

Because of this similarity to methyltransferases and its localization to the nucleus, LaeA/LAE1 has been postulated to regulate transcription by lysine or arginine protein methyltransferase functions [1,29]. However, subsequent work showed that LAE1-modulated expression of genes in $T$. reesei did not correlate with corresponding changes in the histone methylation state at these gene loci [6]. In addition, Patananan et al. [5], using several different experimental approaches failed to identify a protein or other substrate in $A$. nidulans that becomes methylated by LaeA. Instead, LaeA was shown to methylate itself at M207, yet this methylation was not essential for LaeA function, and the corresponding M207 does also not occur in the T. reesei LAE1. All this evidence suggests that LaeA/LAE1 may exert its function by binding to other proteins rather than by methylating histones or other DNA-binding proteins.

LaeA has been isolated because of its role as a regulator of secondary metabolism in A. nidulans [1], and it was therefore surprising that we recently found only a single NRPS to be downregulated in the $\Delta$ lae1 mutant [6]. In the present study, two PKS and one NRPS genes were affected by lae 1 loss-of function, and this effect occurred only at the high growth rate (or was much stronger in the case of the NRPS). The fact that our previous study was performed with lactose grown cells [6] may explain this discrepancy, because growth on lactose is characterized by a very slow growth rate. Thus the effect of LAE1 on secondary metabolism in T. reesei appears to be growth rate dependent, and more pronounced at the high growth rate. Veiga et al. [30] have also found a growth rate-dependency of LaeA and VeA function in Penicillium chrysogenum, although in the opposite direction.

Our data also showed that only a comparatively small percentage of the genes that are affected by LAE1 modulation (i.e. 10\%) are in fact clustered in the genome. This value is much smaller than clustering percentages obtained for gene expression in $T$. reesei under other conditions such as conidiation [24]. While a similar clustering analysis of differently expressed genes in $T$. reesei growing on glycerol and glucose yielded still a lower value (4.3\%; C.P.K., unpublished data), the significance of the $10 \%$ found in this study is unclear. In any case, the data show that most of the genes that are affected by LAE1 loss of function do not appear to be clustered in the genome of T. reesei.

However, the present study revealed a new level at which LaeA/LAE1 may control gene expression: we show that the expression of $50 \%$ of the GCN5-N-acetyltransferases (GNATs) present in the $T$. reesei genome is altered in the Slae1 strain, most of them being downregulated. This effect of LAE1 has not been revealed in previous studies [6], probably due to the use of batch cultures or of different carbon sources. GNATs catalyze the transfer of the acetyl group from acetyl coenzyme A to a primary amine. While - despite of the similarity to the GCN5 protein of the SAGA complex [31] - some of them may thus fulfill metabolic functions, a subgroup of them is known to acetylate histones at specific lysine residues, a process that leads to transcriptional activation and has been implicated in chromatin assembly [32,33]. Unfortunately, none of the $T$. reesei GNATs has so far been functionally investigated, but one - Trire2:120120 - has been shown to be induced on the cellulase inducing carbon sources cellulose [34,35] and lactose [36], is downregulated in T. reesei $\Delta l a e 1$ strain [6] and its overexpression in $T$. reesei leads to a twofold stimulation of cellulase production. This gene is, however, not expressed on glucose and was therefore not detected under the conditions of this work. However, the above noted correlation between LAE1 function and action of the GNAT Trire2:120120 may also be valid for several of the GNATs detected to be LAE1-dependent in this work. In A. nidulans, LaeA function has recently also been linked to histone acetylation: in a multicopy suppressor screen for genes capable of restoring secondary metabolite production in an A. nidulans $\triangle$ laeA mutant, the histone acetyltransferase EsaA was identified [37]. However, EsaA binds to the target histone 
by a chromo-domain and is a member of the Myb_Cef protein family (Pfam 11831), and not a GNAT. Thus, a direct link between GNATs and LaeA/LAE1, which may explain the postulated LaeA-dependent chromatin modification [29], still requires scientific testing. Interestingly, an orthologue of S. cerevisiae SPT10 - an acetyltransferase that directly activates the transcription of histone genes, and whose function is essential for normal cell division [38] - is also strongly downregulated in the T. reesei $\Delta$ lae1 mutant. Its downregulation in $T$. reesei $\Delta l a e 1$ may be a factor contributing to the slower growth observed in this mutant. We should also like to note that the other observed effects such as changes in the growth-rate dependent regulation in the $\Delta$ lae 1 strain would be fully compatible with an action of LAE1 via GNATs, because acetylation by them can also lead to repression of gene expression [39].

Another group of genes that were significantly influenced by LAE1 in this study - but not detected to be affected previously [6] - were amino acid transporters. These transporters can reliably be predicted by the presence of a common structural motif consisting of 12 alpha-helical putative transmembrane segments and cytoplasmically located $\mathrm{N}$ - and C-terminal hydrophilic regions, and belong to the amino acid/polyamine organocation superfamily [40]. In yeasts, they are usually absent during growth on an inorganic nitrogen source but upregulated once organic nitrogen becomes available, using transcriptional [41] and/or posttranscriptional mechanisms [42]. In contrast, the present study shows that these amino acid transporters are expressed in $T$. reesei during growth on an inorganic nitrogen source. These finding is also consistent with the observation that $T$. reesei prefers amino acids as carbon sources when grown in the presence of cellulose or lactose and amino acid mixtures [43], and thus regulation of expression of these permease genes still deserves attention. Interestingly, there is now emerging evidence that amino acid uptake in yeast is regulated by GNAT-dependent histone acetylation [44,45], which would fit to the above supposed role of LAE1 in histone acetylation. We also noted that the effect of LAE1 on amino acid permeases is paralleled by a severe downregulation of a significant number of genes involved in amino acid metabolism in $T$. reesei $\Delta$ lae1 (mainly at the high growth rate), and the above findings of regulation of amino acid uptake by LAE1 can therefore be extended to a general effect on amino acid metabolism. Also, some extracellular proteases and oligopeptide transporters were effected by LAE1 (cf. Additional file 1: Table S1), but these two groups as a whole remained not significantly affected.

Most of the other FunCat groups found to be influenced by LAE1 function were already identified in our earlier study, which used lactose as a carbon source to induce cellulase gene expression [6]. Yet differences in the numbers of genes in the individual groups were noted. It is unclear, however, whether these observations are growth rate- or carbon source-specific.

\section{Conclusions}

In this study, we used growth at controlled growth rates on glucose as a limiting carbon source to study the changes in gene expression effected by the protein methyltransferase LAE1 in $T$. reesei at a genome wide level. The corresponding results confirm several previously detected targets, but also reveal new findings: on one hand, the effect of LAE1 becomes mainly visible upon its deletion whereas overexpression has only a little effect. Also genomic clustering of the lae1-effected transcripts was observed to a much lower extent than previously [6], and is thus either due to the use of glucose as a carbon source or the use of constant growth rates for comparison. However, we detect for the first time that lae1 gene deletion affect the regulation of gene expression by the growth rate. The latter finding likely points to an indirect influence, and suggests that LAE1 influences the formation of both positive as well negative signals or regulators of the respective genes. On the other hand, additional LAE1 targets were obtained of which the GCN5-N-acetyltransferases may offer a new understanding of the mechanism of LAE1 action. Our data show that using $T$. reesei LAE1 as a model - the investigation of the transcriptome in a regulatory mutant at constant growth rates may reveal new insights into the function of the respective gene.

\section{Methods}

\section{Strains and cultivations}

T. reesei QM9414 (ATCC 26921), a moderately cellulaseproducing mutant, and the mutants Slae1 and OElae1 prepared from it [6] was used throughout this work and kept on potato dextrose agar (Sigma, St. Louis, MO).

Chemostat-type continuous cultivations were performed and analyzed as described earlier [17]. Inoculum cultures were pre-grown in $500 \mathrm{ml}$ Erlenmeyer flasks on a rotary shaker (250 r.p.m.) at $30^{\circ} \mathrm{C}$ in a medium described by Karaffa et al. [17] with D-glucose $(10 \mathrm{~g} / \mathrm{L})$ and peptone $(0.1 \mathrm{~g} / \mathrm{L})$ as carbon sources. The feeding medium [17] contained Dglucose at an initial concentration of $3 \mathrm{~g} / \mathrm{L}$, a value low enough to make the culture carbon-limited. Two to four separate steady-states (i.e., independently initiated and run continuous cultures at the constant-mass stage) were sampled and analysed for each dilution rate and fungal strain. Fungal biomass taken from the steady-state chemostat cultures (i. e., from ones that exhibited no changes in biomass dry weight in three successive samples taken over a period of three residence times, and had very little (0.03 $0.05 \mathrm{mM}$ ) residual D-glucose left in the medium during the same period) were subjected to total RNA extraction 
(see below). RNA was subsequently divided for the procedures of the microarray and Real-Time PCR analysis, respectively. This way, mycelia growing under identical environmental (and consequently physiological) conditions were used for the two expression analysis methods.

\section{Analytical methods}

Determination of the mycelial dry weight (DCW) and the assessment of residual glucose concentration in growth media occured as described earlier [46].

\section{Transcriptome analysis}

Mycelia were harvested from steady-state glucose-limited cultures. Total RNAs from cultures with high and low dilution (=specific growth) rate were extracted using SV Total RNA Isolation System (Promega), according to the manufacturer's instructions. cDNA synthesis, labelling and hybridization was performed by Roche NimbleGen (Roche-NimbleGen, Inc., Madison, WI, USA) with a high density oligonucleotide microarray using 60-mer probes representing the 9.129 genes of $T$. reesei as described by Metz et al. [24]. Microarray scanning, data acquisition and identification of probe sets showing a significant difference $(p=0.05)$ in expression level between the different conditions were performed essentially as described previously [24]. Genes were identified by the aid of a completely manually annotated T. reesei genome database proprietary to C.P.K. The Euclidean distance metric method, as implemented in DNASTAR v5.1.2. build 3 (DNAstar Inc., Madison, WI), was used for Hierarchical Clustering. Genes were then classified according to their major annotation in the MIPS Functional Catalogue [33]. To determine whether there were differences in the functional categories in each cluster, the distribution within each cluster was compared to the total distribution of all the annotated genes using independent chi-square tests. The microarray data and the related protocols are available at the GEO web site (www.ncbi.nlm.nih.gov/geo/) under accession number GSE55652.

\section{qPCR}

DNase treated (DNase I, RNase free; Thermo Scientific) total RNA $(1 \mu \mathrm{g})$ was reversely transcribed with the Transcriptor High Fidelity cDNA Synthesis kit (Roche), according to the manufacturer's protocol with a combination (1:1) of the provided oligo-dT and random hexamer primers. Reactions were performed with ready-to-use LightCycler $^{\oplus} 480$ Probes Master mix (Roche), according to the manufacturer's protocol. Primers, amplification efficiency and R-square values are given in Additional file 7: Table S7. Determination of the PCR efficiency was performed using triplicate reactions from a dilution series of cDNA, and the amplification efficiency then calculated from the given slopes in the Realplex v2.2 software. Expression ratios were calculated using REST@ Software [30]. All samples were analyzed in two independent experiments with three replicates in each run.

\section{Analysis of genomic clustering of transcripts}

$T$. reesei genes have not yet been mapped to chromosomes, but their appearance on genomic scaffolds is known. In order to identify whether the transcripts would be clustered to particular areas on these scaffolds, we aligned them onto an ordered list of genes on the individual scaffolds. We tested the presence of clustering of expressed genes by two methods: one employed REEF, a software that identifies genomic regions enriched in specific features, using test statistic based on the hypergeometric distribution applied genome-wide by using a sliding window approach and adopting the false discovery rate for controlling multiplicity [23]. The software, source code and documentation were downloaded from http://telethon.bio.unipd.it/bioinfo/reef/ . To use it for T. reesei, the scaffolds were treated as chromosomes. A window width of $100 \mathrm{~kb}$, a shift of $10 \mathrm{~kb}$ and a threshold q-value of 0.05 were used. A minimum number of 7 and 3 clustered genes were used as a threshold for the analysis of expression at the high and low growth rate, respectively.

\section{Additional files}

\begin{abstract}
Additional file 1: Table S1. Genes differentially regulated in T. reese $\Delta$ lae1 in comparison to the parent strain T. reesei QM 9414.

Additional file 2: Table S2. Genes differentially regulated in T. reesei OElae1 in comparison to the parent strain T. reesei QM 9414

Additional file 3: Table S3. Quantitative expression patterns determined by qRT-PCR of selected genes.

Additional file 4: Table S4. Genes regulated by the growth rate in T. reesei $\Delta$ lae1 in comparison to the parent strain T. reesei QM 9414.
\end{abstract}

Additional file 5: Table S5. Genes regulated by the growth rate in the parent strain T. reesei QM 9414 in comparison to T. reesei $\Delta$ lae1.

Additional file 6: Table S6. Genes regulated in the opposite way in T. reesei $\Delta$ lae1 and the parent strain T. reesei QM 9414

Additional file 7: Table S7. Primers, amplification efficiency and R-square values for the qPCR analysis.

Competing interests

The authors declare that they have no competing interests.

\section{Authors' contributions}

LK, EF and CPK designed and coordinated the study. LK, EF and ZN performed chemostat fermentations and analysis. EF, ÉF and $A O$ carried out samplings, RNA extraction and purification. MP, AS and EF carried out Real-Time PCR analysis. CPK analysed the microarray transcriptome data, performed the annotations and statistics, and interpreted the results. RKA performed the FunCat analysis and prepared the figures. CPK, LK, EF and RKA wrote the manuscript. All authors read and approved the final manuscript.

\section{Acknowledgements}

This study was supported by the Hungarian Scientific Research Fund (OTKA Grant K100660 to EF) and the TÁMOP-4.2.2.A-11/1/KONV-2012-0043 project. Work by CPK and RKA in the Vienna lab was supported by a grant of the 
Austrian Science Foundation to CPK (P 21266). MP was supported by the EU and Hungary, co-financed by the European Social Fund in the framework of the TÁMOP-4.2.4.Av 2-11/1-2012-0001 'National Excellence Program'

\section{Author details}

${ }^{1}$ Department of Biochemical Engineering, Faculty of Science and Technology, University of Debrecen, Egyetem tér 1, H-4032 Debrecen, Hungary. ${ }^{2}$ Institute of Chemical Engineering, University of Technology of Vienna, Gumpendorferstrasse 1a A-1060 Vienna, Austria. ${ }^{3}$ Department of Human Genetics, Faculty of Medicine, University of Debrecen, Nagyerdei krt 98, H-4032 Debrecen, Hungary. ${ }^{4}$ Roche Hungary Ltd., Edison u. 1, H-2040 Budaörs, Hungary. ${ }^{5}$ Austrian Center of Industrial Biotechnology (ACIB), Petersgasse 12, A-8010 Graz, Austria.

Received: 11 March 2014 Accepted: 4 June 2014

Published: 9 June 2014

\section{References}

1. Bok JW, Keller NP: LaeA, a regulator of secondary metabolism in Aspergillus spp. Eukaryot Cell 2004, 3:527-535.

2. McDonagh A, Fedorova ND, Crabtree J, Yu Y, Kim S, Chen D, Loss O, Cairns T, Goldman G, Armstrong-James D, Haynes K, Haas H, Schrettl M, May G, Nierman WC, Bignell E: Sub-telomere directed gene expression during initiation of invasive aspergillosis. PLoS Pathog 2008, 4:e1000154.

3. Perrin RM, Fedorova ND, Bok JW, Cramer RA, Wortman JR, Kim HS, Nierman WC, Keller NP: Transcriptional regulation of chemical diversity in Aspergillus fumigatus by LaeA. PLoS Pathog 2007, 3:e50.

4. Reyes-Dominguez Y, Bok JW, Berger H, Shwab EK, Basheer A, Gallmetzer A, Scazzocchio C, Keller N, Strauss J: Heterochromatic marks are associated with the repression of secondary metabolism clusters in Aspergillus nidulans. Mol Microbiol 2010, 76:1376-1386.

5. Patananan AN, Palmer JM, Garvey GS, Keller NP, Clarke SG: A novel automethylation reaction in the Aspergillus nidulans LaeA protein generates S-methylmethionine. J Biol Chem 2013, 2013(288):14032-14045

6. Karimi-Aghcheh R, Bok JW, Phatale PA, Smith KM, Baker SE, Lichius A, Omann M, Zeilinger S, Seiboth B, Rhee C, Keller NP, Freitag M, Kubicek CP: Functional analyses of Trichoderma reesei LAE1 reveal conserved and contrasting roles of this regulator. G3 (Bethesda) 2013, 3:369-378

7. Bok JW, Balajee SA, Marr KA, Andes D, Nielsen KF, Frisvad JC, Keller NP: LaeA, a regulator of morphogenetic fungal virulence factors. Eukaryot Cell 2005, 4:1574-1582.

8. Sugui JA, Pardo J, Chang YC, Müllbacher A, Zarember KA, Galvez EM, Brinster L, Zerfas P, Gallin Jl, Simon MM, Kwon-Chung KJ: Role of laeA in the regulation of alb1, gliP, conidial morphology, and virulence in Aspergillus fumigatus. Eukaryot Cell 2007, 6:1552-1561.

9. Hoff B, Kamerewerd J, Sigl C, Mitterbauer R, Zadra I, Kürnsteiner H, Kück U: Two components of a velvet-like complex control hyphal morphogenesis, conidiophore development, and penicillin biosynthesis in Penicillium chrysogenum. Eukaryot Cell 2010, 9:1236-1250

10. Wiemann P, Brown DW, Kleigrewe K, Bok JW, Keller NP, Humpf HU, Tudzynski B: FfVel1 and FfLae1, components of a velvet-like complex in Fusarium fujikuroi, affect differentiation, secondary metabolism and virulence. Mol Microbiol 2010, 77:972-994

11. Jiang J, Yun Y, Liu Y, Ma Z: FgVELB is associated with vegetative differentiation, secondary metabolism and virulence in Fusarium graminearum. Fungal Genet Biol 2012, 49:653-662.

12. Wu QX, Jin XJ, Draskovic M, Crews MS, Tenney K, Valeriote FA, Yao XJ, Crews $P$ : Unraveling the numerous biosynthetic products of the marine sediment-derived fungus, Aspergillus insulicola. Phytochem Lett 2012, 5:114-117.

13. Chang PK, Scharfenstein LL, Ehrlich KC, Wei Q, Bhatnagar D, Ingber BF: Effects of laeA deletion on Aspergillus flavus conidial development and hydrophobicity may contribute to loss of aflatoxin production. Fungal Biol 2012, 116:298-307.

14. Kale SP, Milde L, Trapp MK, Frisvad JC, Keller NP, Bok JW: Requirement of LaeA for secondary metabolism and sclerotial production in Aspergillus flavus. Fungal Genet Biol 2008, 45:1422-1429.

15. Amaike S, Keller NP: Distinct roles for VeA and LaeA in development and pathogenesis of Aspergillus flavus. Eukaryot Cell 2009, 8:1051-1060

16. Seiboth B, Karimi RA, Phatale PA, Linke R, Hartl L, Sauer DG, Smith KM, Baker $\mathrm{SE}$, Freitag M, Kubicek CP: The putative protein methyltransferase LAE1 controls cellulase gene expression in Trichoderma reesei. Mol Microbio/ 2012, 84:1150-1164.

17. Karaffa L, Fekete E, Gamauf C, Szentirmai A, Kubicek CP, Seiboth B: D-Galactose induces cellulase gene expression in Hypocrea jecorina at low growth rates. Microbiol-SGM 2006, 152:1507-1514.

18. Portnoy $T$, Margeot $A$, Linke $R$, Atanasova L, Fekete $E$, Sándor $E$, Hartl L, Karaffa L, Druzhinina IS, Seiboth B, Le Crom S, Kubicek CP: The CRE1 carbon catabolite repressor of the fungus Trichoderma reesei: a master regulator of carbon assimilation. BMC Genomics 2011, 12:269.

19. Ilyés H, Fekete E, Karaffa L, Fekete É, Sándor E, Szentirmai A, Kubicek CP: CreA-mediated carbon catabolite repression of $ß$-galactosidase formation in Aspergillus nidulans is growth rate dependent. FEMS Microbiol Lett 2004, 235:147-151.

20. Brown A: Fed-Batch and Continuous Culture. In Fermentation: A Practical Approach. Edited by McNeil B, Harvey LM. Oxford: University Press; 1990:113-130.

21. Jørgensen TR, vanKuyk PA, Poulsen BR, Ruijter GJ, Visser J, Iversen JJ: Glucose uptake and growth of glucose-limited chemostat cultures of Aspergillus niger and a disruptant lacking MstA, a high-affinity glucose transporter. Microbiology 2007, 153:1963-1973.

22. Ruepp A, Zollner A, Maier D, Albermann K, Hani J, Mokrejs M, Tetko I, Güldener U, Mannhaupt G, Münsterkötter M, Mewes HW: The FunCat, a functional annotation scheme for systematic classification of proteins from whole genomes. Nucleic Acids Res 2004, 32:5539-5545.

23. Coppe A, Danieli GA, Bortoluzzi S: REEF: searching REgionally Enriched Features in genomes. BMC Bioinform 2006, 7:453.

24. Metz B, Seidl-Seiboth V, Haarmann T, Kopchinskiy A, Lorenz P, Seiboth B, Kubicek CP: Expression of biomass-degrading enzymes is a major event during conidium development in Trichoderma reesei. Eukaryot Cell 2011, 10:1527-1735

25. David H, Krogh AM, Roca C, Åkesson M, Nielsen J: CreA influences the metabolic fluxes of Aspergillus nidulans during growth on glucose and xylose. Microbiology 2005, 151:2209-2221.

26. Pakula TM, Salonen K, Uusitalo J, Penttilä M: The effect of specific growth rate on protein synthesis and secretion in the filamentous fungus Trichoderma reesei. Microbiology 2005, 151:135-143.

27. Rautio JJ, Smit BA, Wiebe M, Penttilä M, Saloheimo M: Transcriptional monitoring of steady state and effects of anaerobic phases in chemostat cultures of the filamentous fungus Trichoderma reesei. BMC Genomics 2006, 7:247

28. Arvas M, Pakula T, Smit B, Rautio J, Koivistoinen H, Jouhten P, Lindfors E, Wiebe M, Penttilä M, Saloheimo M: Correlation of gene expression and protein production rate - a system wide study. BMC Genomics 2011, 12:616.

29. Reyes-Dominguez Y, Narendja F, Berger H, Gallmetzer A, Fernandez-Martin R, Garcia I, Scazzocchio C, Strauss J: Nucleosome positioning and histone H3 acetylation are independent processes in the Aspergillus nidulans prnD-prnB bidirectional promoter. Eukaryot Cell 2008, 7:656-663.

30. Veiga T, Nijland JG, Driessen AJ, Bovenberg RA, Touw H, van den Berg MA, Pronk JT, Daran JM: Impact of velvet complex on transcriptome and penicillin $\mathrm{G}$ production in glucose-limited chemostat cultures of a $\beta$-lactam high-producing Penicillium chrysogenum strain. OMICS 2012, 16:320-333.

31. Timmers HT, Tora L: SAGA unveiled. Trends Biochem Sci 2005, 30:7-10.

32. Grunstein M: Histone acetylation in chromatin structure and transcription. Nature 1997, 389:349-352.

33. Mizzen CA, Allis CD: Linking histone acetylation to transcriptional regulation. Cell Mol Life Sci 1998, 54:6-20.

34. Bischof R, Fourtis L, Limbeck A, Gamauf C, Seiboth B, Kubicek CP: Comparative analysis of the Trichoderma reesei transcriptome during growth on the cellulase inducing substrates wheat straw and lactose. Biotechnol Biofuels 2013, 6:127.

35. Häkkinen M, Valkonen MJ, Westerholm-Parvinen A, Aro N, Arvas M, Vitikainen M, Penttilä M, Saloheimo M, Pakula TM: Screening of candidate regulators for cellulase and hemicellulase production in Trichoderma reesei and identification of a factor essential for cellulase production. Biotechnol Biofuels 2014, 7:14.

36. Ivanova C, Bååth JA, Seiboth B, Kubicek CP: Systems analysis of lactose metabolism in Trichoderma reesei identifies a lactose permease that is essential for cellulase induction. PLoS One 2013, 8:e62631. 
37. Soukup AA, Chiang YM, Bok JW, Reyes-Dominguez Y, Oakley BR, Wang CC, Strauss J, Keller NP: Overexpression of the Aspergillus nidulans histone 4 acetyltransferase EsaA increases activation of secondary metabolite production. Mol Microbiol 2012, 86:314-330.

38. Chang JS, Winston F: Cell-cycle perturbations suppress the slow-growth defect of spt $10 \Delta$ mutants in Saccharomyces cerevisiae. G3 (Bethesda) 2013, 3:573-583.

39. Bok JW, Soukup AA, Chadwick E, Chiang YM, Wang CC, Keller NP: VeA and MvlA repression of the cryptic orsellinic acid gene cluster in Aspergillus nidulans involves histone 3 acetylation. Mol Microbiol 2013, 89:963-974.

40. Sophianopoulou V, Diallinas G: Amino acid transporters of lower eukaryotes: regulation, structure and topogenesis. FEMS Microbiol Rev 1995, 16:53-75.

41. Godard P, Urrestarazu A, Vissers S, Kontos K, Bontempi G, van Helden J, André $B$ : Effect of 21 different nitrogen sources on global gene expression in the yeast Saccharomyces cerevisiae. Mol Cell Biol 2007, 27:3065-3086.

42. Magasanik B, Kaiser CA: Nitrogen regulation in Saccharomyces cerevisiae. Gene 2002, 290:1-18.

43. Kubicek CP: From cellulose to cellulase inducers: facts and fiction. In Proceedings of the second TRICEL symposium on Trichoderma reesei cellulases and other hydrolytic enzymes. Edited by Suominen P, Reinikainen T. Finland: Foundation of Biotechnical and Industrial Fermentation Research, Espoo; 1993:181-188.

44. Takahashi H, Sun X, Hamamoto M, Yashiroda Y, Yoshida M: The SAGA histone acetyltransferase complex regulates leucine uptake through the Agp3 permease in fission yeast. J Biol Chem 2012, 287:38158-38167.

45. Kaufmann I, White E, Azad A, Marguerat S, Bähler J, Proudfoot NJ: Transcriptional activation of the general amino acid permease gene per1 by the histone deacetylase Clr6 Is regulated by Oca2 kinase. Mol Cell Biol 2010, 30:3396-3410.

46. Fekete E, Karaffa L, Sándor E, Bányai I, Seiboth B, Gyémánt GY, Sepsi A, Szentirmai A, Kubicek CP: The alternative D-galactose degrading pathway of Aspergillus nidulans proceeds via L-sorbose. Arch Microbiol 2004, 181:35-44.

\section{doi:10.1186/1471-2164-15-447}

Cite this article as: Fekete et al:: The transcriptome of lae1 mutants of Trichoderma reesei cultivated at constant growth rates reveals new targets of LAE1 function. BMC Genomics 2014 15:447.

\section{Submit your next manuscript to BioMed Central and take full advantage of:}

- Convenient online submission

- Thorough peer review

- No space constraints or color figure charges

- Immediate publication on acceptance

- Inclusion in PubMed, CAS, Scopus and Google Scholar

- Research which is freely available for redistribution

Submit your manuscript at www.biomedcentral.com/submit 